\title{
Refractive Indices of Five Selected Optical Glasses*
}

\author{
Robert E. Stephens and William S. Rodney
}

\begin{abstract}
The refractive indices of typical samples of five optical glasses made at the National Bureau of Standards were measured over a range of wavelengths from 0.35 to 2.32 microns. This was done in such a way that the values obtained in the visible and invisible portions of the spectrum are consistent with one another. The results are expressed for each glass by a six-constant dispersion formula.
\end{abstract}

Precise data on optical glasses should be readily available in considerable detail in order to satisfy the complex requirements of optical engineers and designers. Only for the visual range of wavelengths are refractive indices satisfactorily available for either domestic or imported glasses, and almost no data are available on spectral transmittances.

The five glasses considered here were made and annealed in the Glass Section at the National Bureau of Standards under the direction of Clarence H. Hahner, who has furnished the weight percentages shown in table 1 , descriptive of their chemical composition as computed from the batch composition. With the exception of the rare-earth glass, these are typical optical glasses and are probably very similar to the corresponding Bausch and Lomb glasses $n=1.517, \quad \nu=64.5 ; n=1.573, \quad \nu=57.4 ; \quad n=$ $1.621, \nu=36.2$; and $n=1.649, \nu=33.8$, as listed in their catalogs under these $D$-line indices and $\nu$ values.

The refractive indices of these glasses were accurately determined in the visible region by means of the high-precision spectrometer made by E. R. Watts

TABLE 1. Chemical compositions of glasses a

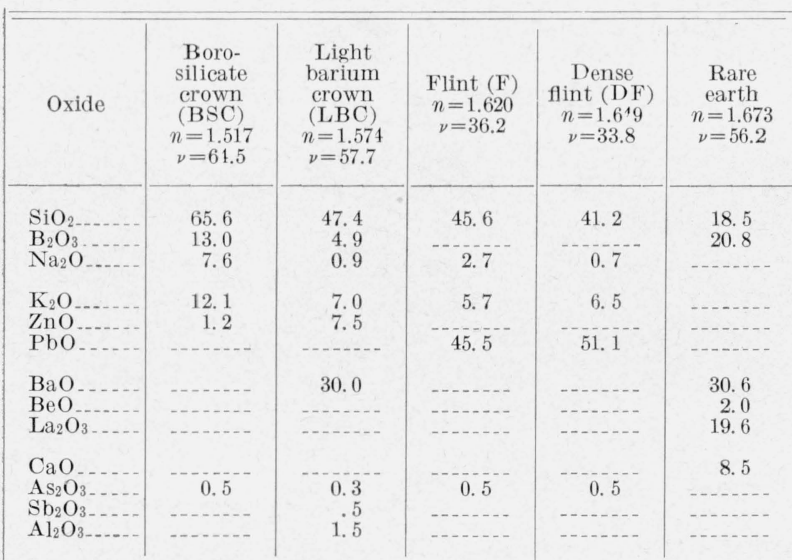

a $n=$ index of refraction for the sodium- $D$ line, and $\nu$, the Abbe value $=(n-1)$ $\left(n_{F}-n_{C}\right)$, where $n_{\mathrm{F}}$ and $n_{\mathrm{C}}$ are refractive indices for the hydrogen $F$ and $C$ lines, $\left(n_{F}-n_{C}\right)$, wh

*The companion papers in this issue present values of the spectral trans*The companion papers in this issue present values of the spectral transmissive properties, densities, and coefficients of linear thermal expansion for
five different types of optical glasses produced by the Optical Glass Section of five different types of optical glasses produced by the Optical Glass Section of the National Bureau of Standards. These measurements have been made in
the different organizational parts of the Bureau appropriate to each type of the different organizational parts of the Bureau appropriate to each type of
measurement, and are, therefore, presented separately. For each type of glass measurement, and are, therefore, presented separately. For each type of glass
the samples have been taken from the same melt, and, therefore, these papers present, collectively, a consistent set of data for the five glasses studied.
\& Son, Ltd., London. ${ }^{1}$ Measurements in the infrared and in the near ultraviolet regions were made on each of two spectrometers. The first was of the Wadsworth mirror type, improvised from a Zeiss monochromator, the same instrument that was used for index determinations on magnesium oxide. ${ }^{2}$ The second was the Gaertner spectrometer equipped with mirrors and a lead sulphide detector, the same apparatus that was used for index determinations on potassium bromide. ${ }^{3}$

The independently observed indices in the infrared and also in the near ultraviolet regions were in fair agreement, and no systematic differences were detected. The values resulting from the second set (Gaertner spectrometer) should be somewhat more accurate because of a potentially higher accuracy in angle measurement, and accordingly much more weight is given to the Gaertner data in computing averages.

The indices of refraction of these glasses were represented by dispersion equations of the form

$$
n^{2}=a^{2}-k \lambda^{2}-p \lambda^{4}+\frac{v}{\lambda^{2}}+\frac{m}{\lambda^{2}-l^{2}}
$$

where $\lambda$ is in microns, and the parameters have appropriate values for the various glasses as given in table 2 . The parameters were adjusted by the NBS Com-

TABLE 2. Parameters of dispersion equations

\begin{tabular}{|c|c|c|c|c|c|}
\hline Parameter & $\begin{array}{c}\text { BSC } \\
n=1.517 \\
\nu=64.5\end{array}$ & $\begin{array}{c}\text { LBC } \\
n=1.574 \\
\nu=57.7\end{array}$ & $\begin{array}{c}\mathbf{F} \\
n=1.620 \\
\nu=36.2\end{array}$ & $\begin{array}{c}\text { DF } \\
n=1.649 \\
\nu=33.8\end{array}$ & $\begin{array}{c}\text { Rare earth } \\
n=1.673 \\
\nu=56.2\end{array}$ \\
\hline $\begin{array}{l}a^{2} \ldots \ldots \\
k \\
p \\
p \\
m_{2}\end{array}$ & $\begin{array}{l}\text { 2. } 2723379 \\
.00961954 \\
.0000982107 \\
.006027086 \\
.004855199 \\
.0237050\end{array}$ & $\begin{array}{l}2.4372255 \\
.00840627 \\
.0000834459 \\
.005289321 \\
.008838177 \\
.0217633\end{array}$ & $\begin{array}{l}2.5551726 \\
.00825919 \\
.0000894995 \\
.010023789 \\
.01340333 \\
.0432000\end{array}$ & $\begin{array}{l}2.6383102 \\
.00832968 \\
.0000939503 \\
.010694553 \\
.01568233 \\
.0451527\end{array}$ & $\begin{array}{l}\text { 2. } 749265 \\
.0109904 \\
.0013455 \\
.0034447 \\
.0146030 \\
.017689\end{array}$ \\
\hline $\begin{array}{l}\text { A verage } \\
\text { residual } \\
\text { ultravi- } \\
\text { olet. } \\
\text { Visible.... } \\
\text { Infrared... }\end{array}$ & $\begin{array}{l}1.9 \times 10^{-5} \\
0.3 \\
1.4\end{array}$ & $\begin{array}{l}0.9 \times 10^{-5} \\
.2 \\
1.0\end{array}$ & $\begin{array}{l}2.6 \times 10^{-5} \\
.3 \\
.7\end{array}$ & $\begin{array}{l}2.2 \times 10^{-5} \\
.8 \\
1.7\end{array}$ & $\begin{array}{l}\text { 1. } 3 \times 10^{-5} \\
.3 \\
1.1\end{array}$ \\
\hline
\end{tabular}

Robert J. Spindler and William S. Rodney, Refractivity of potassium bromide for visible wavelengths, J. Research NBS 49, 253 (1952) RP2361.

2 Robert E. Stephens and Irving H. Malitson, Index of refraction of magne. 2 Robert E. Stephens and Irving H. Malitson, In
sium oxide, J. Research NBS 49,249 (1952) RP2360.

${ }_{3}$ Robert E. Stephens, Earle K. Plyler, William s. Rodney, and Robert J. Spindler, Refractive index of potassium bromide for infrared radiant energy, J. Opt. Soc. Am. 43, 110 (1953). 
TABLE 3. Computed values of refractive indices of glasses

\begin{tabular}{|c|c|c|c|c|c|}
\hline Wavelength & $\begin{array}{c}\text { BSC } \\
n=1.517 \\
\nu=64.5\end{array}$ & $\begin{array}{c}\text { LBC } \\
n=1.574 \\
\nu=57.7\end{array}$ & $\begin{array}{c}\mathrm{F} \\
n=1.620 \\
\nu=36.2\end{array}$ & $\begin{array}{c}\text { DF } \\
n=1.649 \\
\nu=33.8\end{array}$ & $\begin{array}{c}\text { Rare earth } \\
n=1.673 \\
\nu=56.2\end{array}$ \\
\hline $\begin{array}{c}\text { Microns } \\
0.3341 \\
.3404 \\
.3467\end{array}$ & $\begin{array}{l}\text { 1. } 54287 \\
1.54141 \\
1.54001\end{array}$ & 1. 60313 & 1. 67680 & & \\
\hline $\begin{array}{l}.3612 \\
.3650 \\
.3655\end{array}$ & $\begin{array}{l}\text { 1. } 53713 \\
\text { 1. } 53643 \\
\text { 1. } 53635\end{array}$ & $\begin{array}{l}\text { 1. } 59937 \\
1.59846 \\
1.59836\end{array}$ & $\begin{array}{l}\text { 1. } 66870 \\
\text { 1. } 66678 \\
\text { 1. } 66656\end{array}$ & $\begin{array}{l}\text { 1. } 70384 \\
\text { 1. } 70162 \\
\text { 1. } 70136\end{array}$ & $\begin{array}{l}\text { 1. } 70407 \\
\text { 1. } 70295 \\
\text { 1. } 70282\end{array}$ \\
\hline $\begin{array}{l}.3663 \\
.4047 \\
.4078\end{array}$ & $\begin{array}{l}\text { 1. } 53620 \\
\text { 1. } 53044 \\
\text { 1. } 53004\end{array}$ & $\begin{array}{l}1.59816 \\
1.59072 \\
1.59022\end{array}$ & $\begin{array}{l}\text { 1. } 66615 \\
\text { 1. } 65113 \\
\text { 1. } 65014\end{array}$ & $\begin{array}{l}\text { 1. } 70088 \\
\text { 1. } 68360 \\
\text { 1. } 68247\end{array}$ & $\begin{array}{l}\text { 1. } 70259 \\
\text { 1. } 69366 \\
\text { 1. } 69305\end{array}$ \\
\hline $\begin{array}{l}.4340 \\
.4358 \\
.4678\end{array}$ & $\begin{array}{l}\text { 1. } 52709 \\
\text { 1. } 52691 \\
\text { 1. } 52402\end{array}$ & $\begin{array}{l}\text { 1. } 58643 \\
\text { 1. } 58620 \\
1.58253\end{array}$ & $\begin{array}{l}\text { 1. } 64290 \\
\text { 1. } 64247 \\
\text { 1. } 63569\end{array}$ & $\begin{array}{l}\text { 1. } 67421 \\
\text { 1. } 67372 \\
1.66602\end{array}$ & $\begin{array}{l}\text { 1. } 68850 \\
1.68822 \\
1.68380\end{array}$ \\
\hline $\begin{array}{l}.4800 \\
.4861 \\
.5086\end{array}$ & $\begin{array}{l}1.52308 \\
1.52263 \\
1.52112\end{array}$ & $\begin{array}{l}\text { 1. } 58133 \\
\text { 1. } 58076 \\
\text { 1. } 57886\end{array}$ & $\begin{array}{l}\text { 1. } 63352 \\
\text { 1. } 63249 \\
\text { 1. } 62911\end{array}$ & $\begin{array}{l}\text { 1. } 66357 \\
\text { 1. } 66241 \\
\text { 1. } 65860\end{array}$ & $\begin{array}{l}\text { 1. } 68236 \\
\text { 1. } 68167 \\
\text { 1. } 67938\end{array}$ \\
\hline $\begin{array}{l}.5461 \\
.5770 \\
.5791\end{array}$ & $\begin{array}{l}1.51900 \\
\text { 1. } 51754 \\
1.51745\end{array}$ & $\begin{array}{l}1.57620 \\
1.57439 \\
1.57428\end{array}$ & $\begin{array}{l}\text { 1. } 62446 \\
\text { 1. } 62136 \\
1.62116\end{array}$ & $\begin{array}{l}1.65338 \\
1.64990 \\
1.64968\end{array}$ & $\begin{array}{l}\text { 1. } 67618 \\
\text { 1. } 67400 \\
1.67386\end{array}$ \\
\hline $\begin{array}{l}.5893 \\
.6438 \\
.6563\end{array}$ & $\begin{array}{l}1.51702 \\
1.51502 \\
1.51463\end{array}$ & $\begin{array}{l}1.57375 \\
1.57130 \\
1.57082\end{array}$ & $\begin{array}{l}\text { 1. } 62026 \\
\text { 1. } 61617 \\
1.61538\end{array}$ & $\begin{array}{l}\text { 1. } 64867 \\
\text { 1. } 64411 \\
1.64323\end{array}$ & $\begin{array}{l}\text { 1. } 67322 \\
\text { 1. } 67027 \\
\text { 1. } 66969\end{array}$ \\
\hline $\begin{array}{l}.6907 \\
.7065 \\
.7081\end{array}$ & $\begin{array}{l}\text { 1. } 51363 \\
\text { 1. } 51321 \\
\text { 1. } 51317\end{array}$ & $\begin{array}{l}\text { 1. } 56962 \\
\text { 1. } 56911 \\
1.56906\end{array}$ & $\begin{array}{l}\text { 1. } 61342 \\
\text { 1. } 61261 \\
\text { 1. } 61252\end{array}$ & $\begin{array}{l}\text { 1. } 64105 \\
\text { 1. } 64015 \\
1.64006\end{array}$ & $\begin{array}{l}\text { 1. } 66823 \\
\text { 1. } 66762 \\
\text { 1. } 66756\end{array}$ \\
\hline $\begin{array}{r}.7729 \\
1.0140 \\
1.1287\end{array}$ & $\begin{array}{l}1.51166 \\
1.50766 \\
1.50617\end{array}$ & $\begin{array}{l}1.56729 \\
1.56282 \\
1.56128\end{array}$ & $\begin{array}{l}\text { 1. } 60971 \\
\text { 1. } 60310 \\
1.60102\end{array}$ & $\begin{array}{l}\text { 1. } 63695 \\
\text { 1. } 62972 \\
\text { 1. } 62748\end{array}$ & $\begin{array}{l}\text { 1. } 66542 \\
\text { 1. } 66001 \\
\text { 1. } 65812\end{array}$ \\
\hline $\begin{array}{l}1.3570 \\
1,3673 \\
1.3951\end{array}$ & $\begin{array}{l}1.50341 \\
1.50328 \\
1.50295\end{array}$ & $\begin{array}{l}1.55859 \\
1.55847 \\
1.55816\end{array}$ & $\begin{array}{l}1.59767 \\
1.59754 \\
1.59717\end{array}$ & $\begin{array}{l}1.62394 \\
1.62380 \\
1.62342\end{array}$ & $\begin{array}{l}1.65483 \\
1.65468 \\
1.65430\end{array}$ \\
\hline $\begin{array}{l}1.5295 \\
\text { 1. } 6932 \\
1.7092\end{array}$ & $\begin{array}{l}\text { 1. } 50132 \\
\text { 1. } 49925 \\
1.49905\end{array}$ & $\begin{array}{l}1.55666 \\
1.55480 \\
1.55461\end{array}$ & $\begin{array}{l}1.59546 \\
1.59343 \\
1.59323\end{array}$ & $\begin{array}{l}1.62164 \\
1.61955 \\
1.61935\end{array}$ & $\begin{array}{l}1.65245 \\
1.65009 \\
1.64992\end{array}$ \\
\hline $\begin{array}{l}\text { 1. } 8131 \\
1.9701 \\
\text { 2. } 2493 \\
2.3254\end{array}$ & $\begin{array}{l}\text { 1. } 49766 \\
\text { 1. } 49544 \\
\text { 1. } 49108 \\
\text { 1. } 48979\end{array}$ & $\begin{array}{l}\text { 1. } 55338 \\
\text { 1. } 55145 \\
1.54770 \\
1.54659\end{array}$ & $\begin{array}{l}1.59193 \\
1.58992 \\
1.58611 \\
1.58501\end{array}$ & $\begin{array}{l}1.61802 \\
\text { 1. } 61599 \\
1.61214 \\
1.61103\end{array}$ & $\begin{array}{l}1.64839 \\
1.64598 \\
1.64128 \\
1.63989\end{array}$ \\
\hline
\end{tabular}

putation Laboratory, using the method of least squares. The magnitude and distribution of the plus and minus residuals are such that the formulas are unquestionably adequate for representing the data. The computed values, which are adjudged to be better than the observed, values are presented in table 3.

The necessity of using a dispersion equation with six constants was not anticipated. Trials with four carefully adjusted constants, however, proved inadequate in that for the first four glasses there were evident systematic differences between the observed and the computed indices. Such systematic failures for four-constant formulas were as large as 5 or $10 \times 10^{-5}$ outside the visible region, and the deviations were more convincing in the infrared because of more observed points there than in the violet. Moreover, the character of the systematic failures was similar for the different glasses, and thus the equation was considered inadequate in form.

The addition of a term in $\lambda^{4}$ was obviously a suitable expedient for the infrared and one in $1 / \lambda^{2}$ improved the ultraviolet end, and both seemed necessary. The resulting residuals are all less than $\pm 5 \times 10^{-5}$, with the exception of 2 (of opposite sign) in the ultraviolet and, similarly, 2 in the infrared. One-half of all residuals are smaller than $\pm 0.7 \times 10^{-5}$. The remaining systematic trends between observed and computed values are not over \pm 1 or $2 \times 10^{-5}$.

Washington, November 19, 1953. 